\title{
Qualitative and Quantitative Assessment of Patient and Carer Experience of Chemotherapy (Docetaxel) in Combination with Androgen Deprivation Therapy (ADT) for the Treatment of Metastatic Hormone- Sensitive Prostate Cancer (mHSPC)
}

Tetsuro Ito (D) · Laura Grant • Bryony R. Duckham • Amanda J. Ribbands •

Adam Gater (D)

Received: August 21, 2018 / Published online: November 10, 2018

(C) The Author(s) 2018

\begin{abstract}
Introduction: Recent studies suggest that docetaxel plus androgen deprivation therapy can prolong survival among men with metastatic hormone-sensitive prostate cancer (mHSPC). However, as a cytotoxic therapy, there is a need to understand the experiences of men with mHSPC receiving docetaxel and their carers in a real-world setting.

Methods: During phase 1, semi-structured qualitative interviews were conducted with men with mHSPC $(n=31)$ and their carers $(n=14)$ in Europe to elicit in-depth data concerning their experiences with docetaxel. Eighteen men were also asked to record their experiences in a diary for 7 days. During phase 2 , men with mHSPC $(n=161)$ and carers of men with mHSPC $(n=135)$ completed an online survey comprising self-report questionnaires including
\end{abstract}

Enhanced digital features To view enhanced digital features for this article go to https://doi.org/10.6084/ m9.figshare.7228892.

T. Ito

Janssen Health Economics and Market Access EMEA,

High Wycombe, UK

L. Grant · A. Gater $(\bowtie)$

Patient-Centered Outcomes, Adelphi Values,

Cheshire, UK

e-mail: adam.gater@adelphivalues.com

B. R. Duckham · A. J. Ribbands

Adelphi Research UK, Cheshire, UK the Cancer Therapy Satisfaction Questionnaire, Brief Fatigue Inventory, Functional Assessment of Cancer Therapy-Prostate, EuroQol-5-Dimensions and the Burden Scale for Family Caregivers (carers only).

Results: At the outset of therapy, men reported a willingness to take docetaxel to prolong their life, despite being fearful of the potential side effects and impacts on their daily lives. Patient and carer experiences were generally consistent with pre-treatment expectations. However, variations in individual experiences and their ability to tolerate side effects were evident. Fatigue emerged as a prominent symptom with the majority ( $n=98,60.9 \%$ ) of men reporting experiencing moderate-severe fatigue in the past $24 \mathrm{~h}$. Participant ratings of fatigue were strongly correlated with health-related quality of life $(r=-0.82)$. Nausea, diarrhoea and sore mouth were also among the most bothersome symptoms for participants.

Conclusions: Findings from this study highlight that real-world experience of docetaxel may differ from that observed in clinical trials and that care must be taken to ensure that treatment options are tailored to the needs of individual patients to promote not only how long patients survive but also the quality of that survival.

Funding: Janssen 
Keywords: Carers; Docetaxel; Health-related quality of life; Metastatic hormone-sensitive prostate cancer; Treatment satisfaction

\section{INTRODUCTION}

Prostate cancer is the most common cancer in men in Europe [1]. Approximately $17-34 \%$ of men in the UK diagnosed with prostate cancer have a metastatic form of the disease at diagnosis [1]. The androgen receptor is involved in the growth and spread of prostate cancer and can initially be treated hormonally using androgen deprivation therapy (ADT). Men with metastatic prostate cancer who have not been previously treated with ADT or who are still sensitive to ADT and not castration resistant are defined as having mHSPC.

Evidence from recent studies has highlighted that administration of docetaxel (chemotherapy) plus ADT among men with mHSPC can prolong overall survival, particularly among those men with 'high-risk' forms of the disease and generally poorer prognosis [2-7]. Accordingly, the most recent European Association of Urology (EAU) and European Society for Medical Oncology (ESMO) guidelines recommend that docetaxel combined with ADT should be considered the standard of care for men with metastases at first presentation, provided they are fit enough to receive the drug $[8,9]$. To date, however, there has been little exploration of the experiences and perspectives of men with mHSPC receiving docetaxel, particularly in terms of treatment satisfaction and Health-Related Quality of Life (HRQoL). The aim of this study, therefore, was to explore the perspectives of men and carers of men with mHSPC who had received docetaxel using qualitative and quantitative research methods.

\section{METHODS}

\section{Design}

A cross-sectional, multinational mixed methods study was conducted in two phases. Phase 1 (qualitative stage) involved the conduct of semistructured interviews among men with mHSPC and who had experience of docetaxel and informal (unpaid) carers of friends or relatives with mHSPC and who had been treated with docetaxel. Phase 2 comprised a quantitative survey among men with mHSPC and carers of men with mHSPC to quantify experiences with docetaxel.

All procedures performed in studies involving human participants were in accordance with the ethical standards of the institutional and/or national research committee and with the 1964 Helsinki Declaration and its later amendments or comparable ethical standards. Informed consent was obtained from all participants for each stage of the study and ethical approval was granted by Freiburger Ethik-Kommission International (study code: 22894, FEKI code 016/1630).

\section{Participants}

Participants for both phases of the research were recruited from five European countries (France, Germany, UK, Italy and Spain) between October 2016 and January 2017 (qualitative phase 1) and September 2017 and March 2018 (quantitative phase 2).

To be eligible for participation in either phase of the study, men with mHSPC were required to: have a formal diagnosis of mHSPC; have metastases outside of the pelvis and have received at least two cycles of docetaxel within the past 6 months. During the qualitative phase, a small number of men with mHSPC $(n=5)$ who had refused docetaxel following discussions with their healthcare professional were also included.

Only primary carers of men with mHSPC were eligible for participation in the study. For the purposes of this study, primary carers were defined as friends or relatives that visited at least twice weekly, carried out tasks to help the patient on a regular basis and/or attended several meetings where the patient had met their health care professional. Formal (paid) carers were excluded from participation.

\section{Phase 1: Qualitative Interviews}

Semi-structured qualitative interviews were conducted with men with mHSPC $(n=31)$ and carers 
of men with mHSPC $(n=14)$ to elicit in-depth data concerning their experiences of docetaxel. Initially men were asked to talk openly about their experience of mHSPC and their medical history. The next section of the interview focused on the men's experience of previous treatments, treatment expectations and their journey from diagnosis to the current day. To support discussion of the treatment journey, participants were provided with a blank depiction of a timeline on which they were asked to draw a line depicting their highs and lows, which were to be defined in any way that the participant saw relevant. The final part of the interview asked patients to reflect on the impact that their diagnosis and treatment had on daily life. The carer interview was similar in structure. All interviews were conducted in the local language by trained qualitative interviewers.

In addition to the interviews, a subset of the men recruited to participate in the interview $(n=18)$ were also asked to complete a diary for a period of 7 days to probe on their experiences and how they were feeling each day.

Data from the semi-structured interviews and diaries were analysed using thematic analysis techniques centered upon the grouping of verbatim quotes.

\section{Phase 2: Quantitative Stage}

Eligible participants completed a 30-min online survey at home. The content of the survey was informed by the findings from the phase 1 qualitative interviews. At the beginning of the survey, participants were asked to provide background demographic and clinical information as well as answer a series of screening questions to determine eligibility. Participants were then asked to complete a series of diseasespecific and generic patient-reported outcome (PRO) questionnaires designed to collect information concerning treatment satisfaction and HRQoL:

- Cancer Therapy Satisfaction Questionnaire (CTSQ): The CTSQ is a patient-completed questionnaire developed and validated for use in various cancers to evaluate satisfaction with intravenous and/or oral chemotherapy, biological and hormonal therapies. The CTSQ comprises 16 items measuring 3 domains; expectations of therapy (ET), feelings about side effects (FSE) and satisfaction with therapy (SWT). Each domain is scored from 0 to 100 with higher scores representing greater satisfaction [10].

- The Brief Fatigue Inventory (BFI): The BFI comprises nine items used to assess the severity and impact of cancer-related fatigue. All items are rated on a scale from 0 (no fatigue/does not interfere) to 10 (fatigue as bad as you can imagine/completely interferes). Eight of the nine items use a recall period of the 'past $24 \mathrm{~h}$ ' and one item assesses fatigue 'right now'. A global fatigue score can be calculated as an average of all item responses [11].

- Functional Assessment of Cancer TherapyProstate (FACT-P): The FACT-P is a multidimensional, self-report QoL instrument specifically designed for use with prostate cancer patients. The questionnaire consists of 27 core items assessing four domains, including physical well-being (PWB), social/family well-being (S/FWB), emotional well-being (EWB) and functional well-being (FWB), and a prostate cancer-specific (PCS) subscale (12 items). Each item is rated on a 0-4 Likert type scale ranging from 'not at all' to 'very much'. Item scores are summed to produce domain scores for which the range varies by domain. Higher scores represent better health and HRQoL [12].

- EuroQol-5-Dimensions 5-Levels (EQ-5D-5L): The EQ-5D-5L is a standardized questionnaire applicable to a wide range of health conditions and treatments and requires respondents to rate their health status in terms of "Mobility", "Self-Care", "Usual Activities", "Pain/Discomfort" and "Anxiety/Depression" on a 5-point response continuum (1-no problem to 5-extreme problem/unable to do) with lower scores representing better health. A 'utility index score' can be calculated for each patient ranging from -0.59 to 1.00 with 1.00 indicating 'full health'. In addition, respondents rate their health status on a $100-\mathrm{mm}$ visual analogue scale [13].

Carers were also asked to provide demographic and clinical information about 
themselves and the patient they care for and asked to complete the Burden Scale for Family Caregivers (BSFC). The BSFC comprises 28 items designed to assess the impact on emotional and physical health of family caregivers. All items are rated on a 4-point Likert scale with response options ranging from 0 (strongly disagree) to 3 (strongly agree) [14].

The data from participants were entered into a database for analysis. The database was developed with quality control functionalities in place (e.g., validation alerts when out-ofrange values are entered, auto-tabbing, spell checking, shortcut keys, data entry status flags). Participant scores per item and summary scores for each questionnaire were calculated in accordance with developer instructions. For men with $\mathrm{mHSPC}$, the relationship between PRO scores was explored via the calculation of Pearson correlation coefficients.

\section{RESULTS}

\section{Phase 1: Qualitative Interviews}

\section{Demographic and Clinical Characteristics}

Interviews were conducted with a total of 31 men with mHSPC, including 26 with experience of docetaxel and 5 participants who had no experience of docetaxel having decided against taking it for the treatment of their mHSPC. A further 14 interviews were conducted with primary carers of men with mHSPC. Key demographic and clinical characteristics of participants are summarized in Table 1.

\section{Expectations Prior to Docetaxel Treatment}

At the outset of therapy, men reported a willingness to take docetaxel to prolong their life despite being fearful of the potential side effects and impacts on their daily lives. Men were particularly concerned about the range of side effects (particularly the side effects that have a more physical or psychological impact), the way the treatment may impact on their lifestyle and the way the treatment may affect their family relationships and employment.
"I was mostly concerned about the nausea and vomiting, and I also heard about the weight loss and loss of mobility".

"For me a huge worry were the side effects; it is already difficult to live with this disease; I already have to accept a change in lifestyle".

Patients expected the treatment to: reduce the size of the tumour, prevent cancer from spreading/slow down spreading and ultimately give patients longer time to live.

"I said I'm willing to take the risks of having chemotherapy to see what benefits it has to $m e^{\prime \prime}$.

"I said yes, whatever it takes. I want to get through this".

\section{Experiences During Therapy}

The majority of men reported experiencing benefits associated with docetaxel, evident by reduced levels of prostate-specific antigen (PSA) in their blood. The benefits included: PSA reduction, progression slowed/controlled, feeling positive after hospital appointments, no worsening of condition and maintained some independence. However, a wide range of side effects and impacts on daily life were reported, such as nausea, diarrhoea, fatigue and others. The most burdensome side effects reported by patients included fatigue, nausea and hair loss as well as other side effects including: sore mouth, mouth ulcers, dry lips, nose bleeds, anaemia, feeling bloated, bone pain, bruising, nail loss and rash. Variations in individual experiences and patients' ability to tolerate side effects were evident.

Patients reported a range of impacts associated with docetaxel, including: impact on daily activities (e.g., unable to walk as quickly, unable to participate in sports/leisure activities, limited amount of work), impact on social interaction (e.g., limit visitors because of the risk of infection, avoid social activities) and psychological and emotional impacts (e.g., different outlook on life, anger at the way their life has changed, more volatile moods due to treatment).

Consideration of individual participant experiences and treatment journeys highlighted 
Table 1 Participant clinical and demographic characteristics

\begin{tabular}{|c|c|c|c|c|}
\hline \multirow[t]{2}{*}{ Variable } & \multicolumn{2}{|l|}{ Phase 1: qualitative } & \multicolumn{2}{|l|}{ Phase 2: quantitative } \\
\hline & $\begin{array}{l}\text { Men with mHSPC } \\
(n=31)\end{array}$ & $\begin{array}{l}\text { Carers } \\
(n=14)\end{array}$ & $\begin{array}{l}\text { Men with mHSPC } \\
(n=161)\end{array}$ & $\begin{array}{l}\text { Carers } \\
(n=135)\end{array}$ \\
\hline \multicolumn{5}{|l|}{ Country, $n(\%)$} \\
\hline France & $5(16.1)$ & $3(21.4)$ & $16(9.9)$ & $9(6.7)$ \\
\hline Germany & $2(6.5)$ & $2(14.3)$ & $55(34.2)$ & $40(29.6)$ \\
\hline Italy & $10(32.3)$ & $4(28.6)$ & $48(29.8)$ & $38(28.1)$ \\
\hline Spain & $10(32.3)$ & $4(28.6)$ & $12(7.5)$ & $16(11.9)$ \\
\hline UK & $4(12.9)$ & $1(7.1)$ & $30(18.6)$ & $32(23.7)$ \\
\hline \multicolumn{5}{|l|}{ Age in years, $n(\%)$} \\
\hline$\leq 40$ & $0(0.0)$ & $1(7.1)$ & $6(3.7)$ & $14(10.4)$ \\
\hline $41-50$ & $1(3.2)$ & $4(28.6)$ & $8(5.0)$ & $39(28.9)$ \\
\hline $51-60$ & $11(35.5)$ & $5(35.7)$ & $30(18.6)$ & $29(21.5)$ \\
\hline $61-70$ & $11(35.5)$ & $2(14.3)$ & $64(39.8)$ & $35(25.9)$ \\
\hline $71-80$ & $8(25.8)$ & $2(14.3)$ & $50(31.1)$ & $18(13.3)$ \\
\hline$\geq 81$ & $0(0.0)$ & $0(0.0)$ & $3(1.9)$ & $0(0.0)$ \\
\hline \multicolumn{5}{|l|}{ Working status, $n(\%)$} \\
\hline Working full time & $7(22.6)$ & $4(28.6)$ & $16(9.9)$ & $36(26.7)$ \\
\hline Working part time & $1(3.2)$ & $3(21.4)$ & $10(6.2)$ & $22(16.3)$ \\
\hline Self-employed & $3(9.7)$ & $2(14.3)$ & $9(5.6)$ & $6(4.4)$ \\
\hline Currently not working & $1(3.2)$ & $2(14.3)$ & $20(12.4)$ & $23(17.0)$ \\
\hline Retired & $17(54.8)$ & $3(21.4)$ & $106(65.8)$ & $45(33.3)$ \\
\hline Other & $2(6.5)$ & $0(0.0)$ & $0(0.0)$ & $3(2.2)$ \\
\hline \multicolumn{5}{|l|}{ Cycles of docetaxel, $n(\%)$} \\
\hline 0 cycles (refusers) & $5(16.1)$ & - & - & - \\
\hline $2-4$ cycles & $14(45.2)$ & $9(64.3)$ & $99(61.5)$ & $91(67.4)$ \\
\hline $5-6$ cycles & $10(32.3)$ & $4(28.6)$ & $39(24.2)$ & $33(24.4)$ \\
\hline More than 6 cycles & $2(6.5)$ & $1(7.1)$ & $23(14.3)$ & $11(8.1)$ \\
\hline \multicolumn{5}{|l|}{ Classification of mHSPC, $n$ (\%) } \\
\hline Very aggressive cancer ${ }^{a}$ & & & $114(70.8)$ & $103(76.3)$ \\
\hline $\begin{array}{l}\text { Bone metastases in } 3 \text { or more } \\
\text { places }^{\mathrm{a}}\end{array}$ & & & $127(78.9)$ & $108(82.4)$ \\
\hline $\begin{array}{l}\text { Metastases within internal } \\
\text { organs }^{\mathrm{a}}\end{array}$ & & & $97(60.2)$ & $66(50.4)$ \\
\hline
\end{tabular}


Table 1 continued

\begin{tabular}{|c|c|c|c|c|}
\hline \multirow{2}{*}{ Variable } & \multicolumn{2}{|l|}{ Phase 1: qualitative } & \multicolumn{2}{|l|}{ Phase 2: quantitative } \\
\hline & $\begin{array}{l}\text { Men with mHSPC } \\
(n=31)\end{array}$ & $\begin{array}{l}\text { Carers } \\
(n=14)\end{array}$ & $\begin{array}{l}\text { Men with mHSPC } \\
(n=161)\end{array}$ & $\begin{array}{l}\text { Carers } \\
(n=135)\end{array}$ \\
\hline None of the above & & & $5(3.1)$ & $4(3.1)$ \\
\hline Not specified & & & $15(9.3)$ & $7(5.3)$ \\
\hline
\end{tabular}

a Criteria used to define participants as 'high risk'. Note: multiple categories could be selected

that the experience and associated impact of docetaxel for each patient are different. However, analysis of these journeys identified four broad subtypes (Fig. 1).

Investigation of participant day-to-day experience via completion of the 7-day daily diary revealed that 'good days' are characterized by being able to complete household activities (e.g., cleaning and shopping) and activities outside the home with family (e.g., shopping). In contrast, bad days are characterized by feelings of fatigue, pain and nausea limiting their ability to complete household tasks or activities outside of the house (Fig. 2).
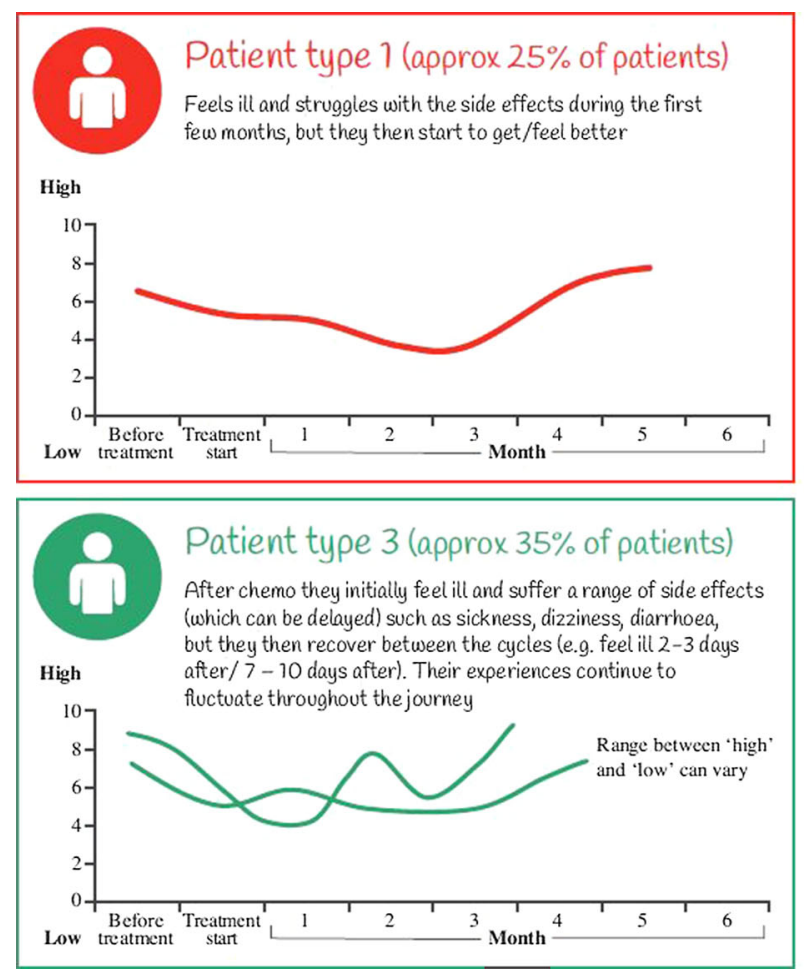

\section{Carer Experience}

Carers felt it was their role to care for the patient and reported adapting their lives to meet the needs of the patient, including: re-arranging home life, taking time off work and changing activities to ones more suitable to the patient.

"I monitor him all the time. I start from the idea that it is my responsibility".

Carers were also negatively impacted by docetaxel treatment, despite their efforts to stay positive and support the patients. Carers specifically mentioned the emotional impact of witnessing a family member battle against
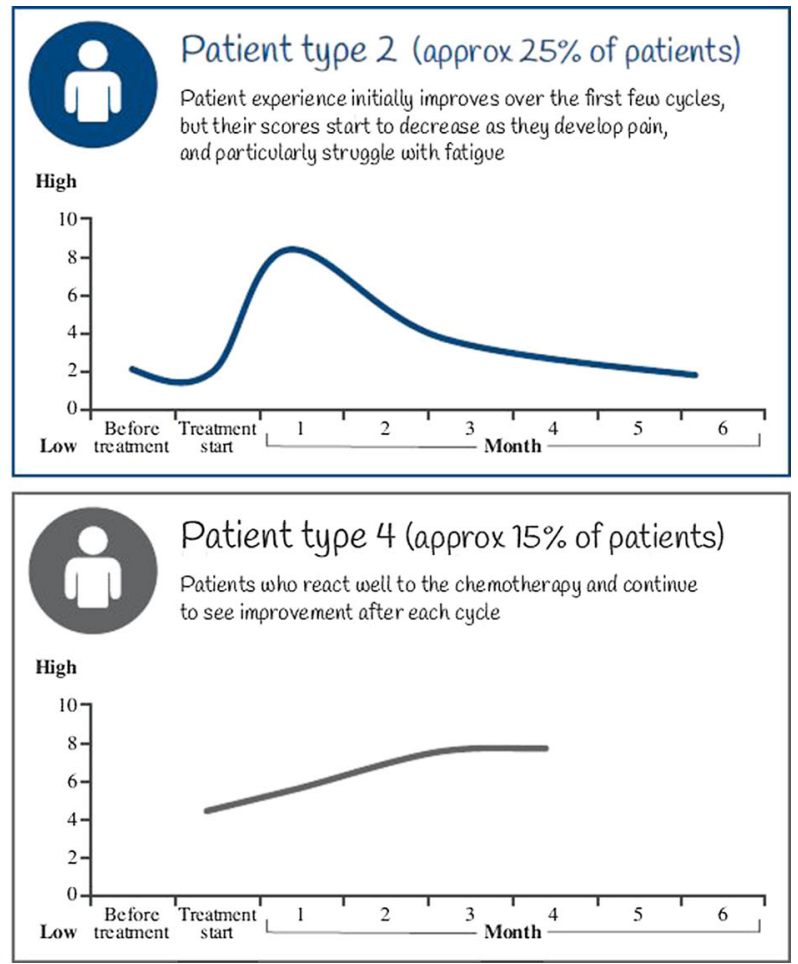

Fig. 1 Experiences and personal journeys (high vs. low points) of men with mHSPC from decision to start treatment with chemotherapy (docetaxel): select examples 
a

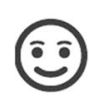

(2)

2.

b

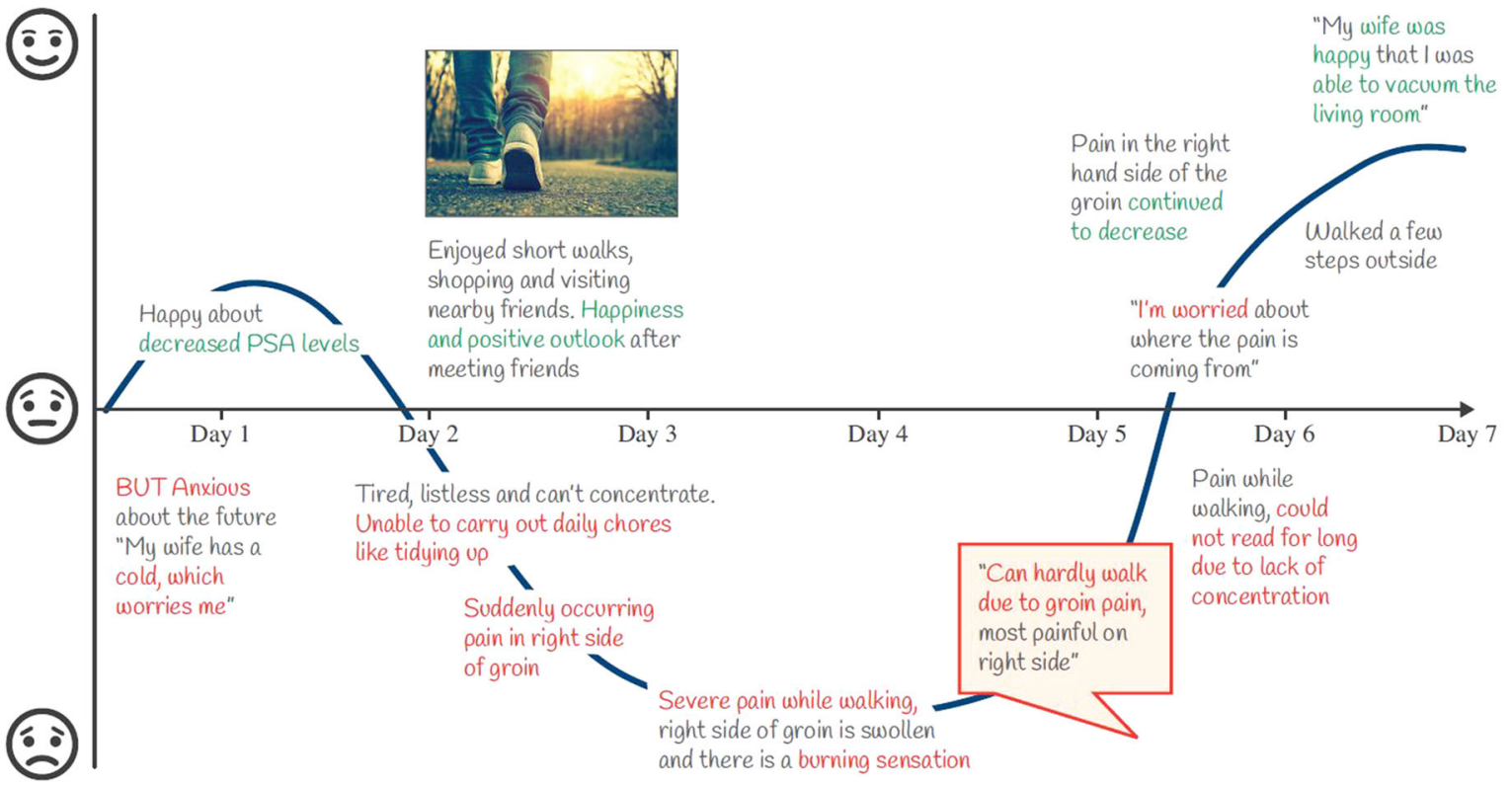

"Today I feel better I I I what I like doing"

than previous days"

"Went to the shops near home with my daughter and bought bread "Feeling better and quite well, in the afternoon I went out with my wife and we did some shopping"

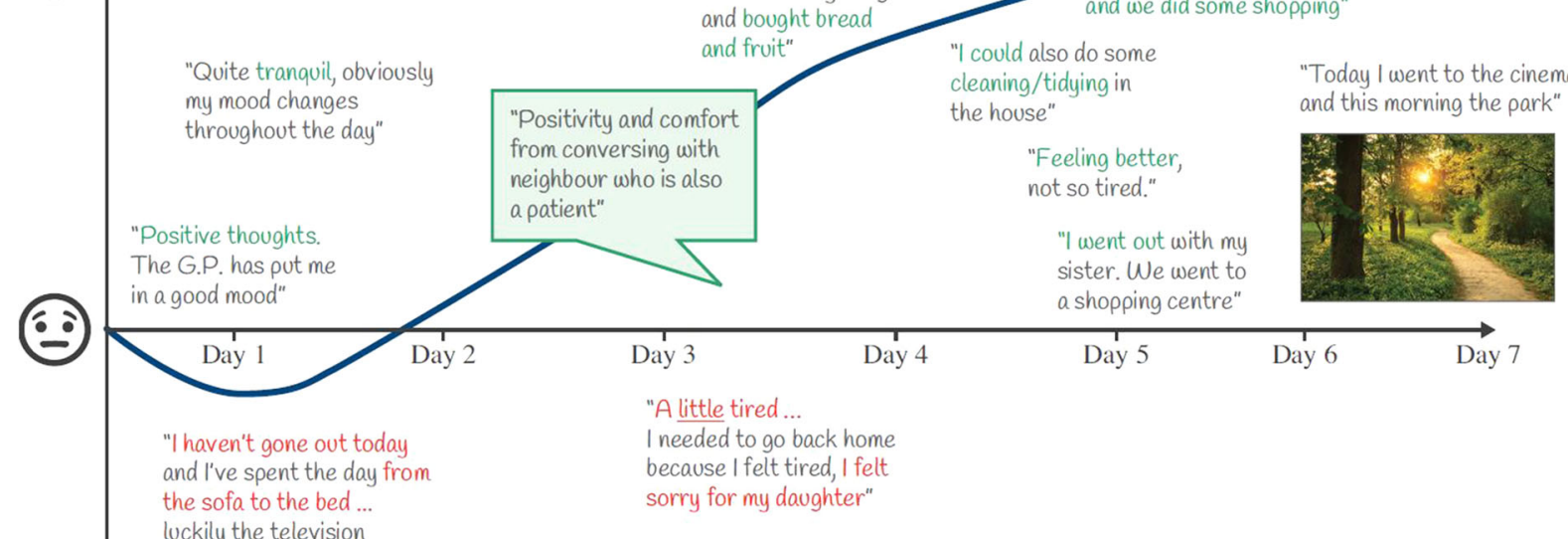

luckily the television

keeps me company"

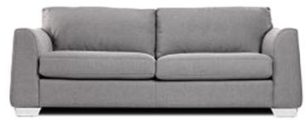

because I felt tired, I felt

sorry for my daughter"
"I could also do some

cleaning/tidying in

"Feeling better not so tired."

went out with m ster. We went to and this morning the park" 


\section{Reasons Why mHSPC Patients Refuse Docetaxel}

Those refusing to take docetaxel said that their decision was driven by what they had seen of and heard from other patients who had previously received docetaxel. Docetaxel was viewed as a last resort treatment.

"I first became aware of chemo through patients in support group. Saw that they were not doing well during chemotherapy".

"Many who had been in chemotherapy said they wouldn't do it again".

"Chemotherapy would only be an option for $m e$ as the very last resort, if nothing else helps anymore".

\section{Phase 2: Quantitative stage}

\section{Demographic and Clinical Characteristics}

A total of 161 men with mHSPC and 135 carers of men with mHSPC completed the quantitative survey. Of note, most of these participants $(n=141 ; 88.2 \%)$ were categorised as having 'high-risk' disease. Demographic and clinical characteristics are presented in Table 1.

\section{Experiences and Perspectives of Men with $\mathrm{MHSPC}$}

CTSQ scores among men with mHSPC indicated that participants were generally satisfied with their current cancer therapy. Mean scores among men were highest for the CTSQ domain of 'satisfaction with therapy' (mean 72.0, SD 16.0), followed by the 'expectations of therapy' domain (mean 64.1, SD 17.0), and the lowest mean scores (indicating lower satisfaction) were associated with the 'feelings about side effects' domain (mean 47.2, SD 19.1) (Table 2).

Consideration of mean scores by participant age highlighted that scores for the CTSQ 'expectations of therapy' and 'satisfaction with therapy' domains generally decreased with increasing age. For example, mean 'expectations of therapy' scores were 77.5 for participants aged $41-50$ years $(n=8)$ compared with 61.5 for participants aged older than 71 years $(n=53)$. Similarly, mean 'satisfaction with therapy' scores were 79.4 for participants aged
Table 2 Patient-reported outcome results among men with $\operatorname{mHSPC}(n=161)$

\begin{tabular}{ll}
\hline PRO score & Mean (SD) \\
\hline CTSQ (0-100) & \\
Expectations of therapy & $64.1(17.0)$ \\
Feelings about side effects & $47.2(19.1)$ \\
Satisfaction with therapy & $72.0(16.0)$ \\
BFI (0-10) & \\
Global score & $4.3(2.7)$ \\
Fatigue right now & $4.7(2.7)$ \\
Usual level of fatigue during past 24h & $4.6(2.6)$ \\
Worst level of fatigue during past 24 h & $5.1(2.7)$ \\
Interference during the past 24h on; & \\
General activity & $4.3(3.1)$ \\
Mood & $3.9(3.2)$ \\
Walking ability & $3.9(3.2)$ \\
Normal work & $4.3(3.2)$ \\
Relations with other people & $3.6(3.0)$ \\
Enjoyment of life & $4.1(3.2)$ \\
FACT-P & \\
Total Score (0-156) & $92.4(21.9)$ \\
Physical well-being domain (0-28) & $17.6(6.9)$ \\
Social well-being domain (0-28) & $13.8(10.2)$ \\
Emotional well-being domain $(0-24)$ & $15.0(5.7)$ \\
Functioning well-being domain (0-28) & $14.1(7.1)$ \\
Prostate cancer-specific subscale (0-48) & $27.3(8.5)$ \\
EQ-5D-5L & \\
VAS (0-100) & \\
Index value (0-1) & \\
\hline
\end{tabular}

41-50 years $(n=8)$ compared with 68.0 for participants aged older than 71 years $(n=53)$. Scores for the CTSQ 'feelings about side effects' domain were relatively consistent across age groups. 
Reports among men with mHSPC highlighted hair loss/thinning $(n=118,73.3 \%)$, fatigue $(n=111,68.9 \%)$ and nausea and sickness $(n=97$, $60.2 \%$ ) to be the most commonly experienced treatment-related side effects (Table 3). However, despite being the most common treatment-related side effect, hair loss/thinning was reported by participants to be the least bothersome of reported side effects (mean $=1.5, \mathrm{SD}=1.3$ ). Those treatment-related side effects reported to be the most bothersome among participants included nausea and sickness (mean $=2.9, \mathrm{SD}=1.0$ ), diarrhoea $($ mean $=2.9, \mathrm{SD}=1.1)$, mouth ulcers/sore mouth (mean $=2.8 \mathrm{SD}=1.2)$ and fatigue (mean $=2.7, \mathrm{SD}=1.0$ )

Two-thirds of the men $(61.5 \%, 99 / 161)$ reported feeling unusually tired or fatigued in the last week. Ratings of worst fatigue indicated that the majority of men with mHSPC had experienced moderate-severe fatigue $(n=98$, $60.9 \%$ ) in the past $24 \mathrm{~h}$. Fatigue was reported by participants to interfere the most with general levels of activity and normal work (mean 4.3, SD 3.1) (Table 2).

Observation of scores derived from the FACT-P did not reveal deficits in specific aspects of HRQoL (Table 2). EQ-5D-5L self-rated health status (VAS) and index values were both lower than those from the general population (82.4 and 0.86, respectively), indicating poorer HRQoL [15] (Table 2). Based on the proportion of participants selecting responses to individual items of the EQ-5D-5L, the most common issue for participants was pain/discomfort with problems reported by 130 participants $(80.7 \%)$. Problems with self-care were reported least frequently by participants but were still reported by 67 participants (41.6\%) (Fig. 3).

Strong correlations $(r=-0.82)$ were observed between global scores for the BFI and FACT-P, with those participants with higher levels of fatigue reporting lower levels of HRQoL. Moderate correlations $(r=0.47-0.66)$ were observed between participant ratings of feelings about side effects and satisfaction with therapy and reported levels of fatigue (BFI), HRQoL (FACT-P) and health status (EQ-5D-5L VAS). Similarly, moderate correlations $(r=-0.46$ to 0.63$)$ were also observed between participant ratings of satisfaction with therapy and reported levels of fatigue
Table 3 Treatment-related side effects reported by men with $\operatorname{mHSPC}(n=161)^{\mathrm{a}}$

\begin{tabular}{|c|c|c|}
\hline Side effects & $\begin{array}{l}\text { Participants } \\
\text { reporting } \\
\text { experiencing side } \\
\text { effect, } n(\%)\end{array}$ & $\begin{array}{l}\text { Mean participant } \\
\text { rating of bother } \\
\text { (0-4: not } \\
\text { bothered-very } \\
\text { bothered) }\end{array}$ \\
\hline $\begin{array}{l}\text { Hair loss/ } \\
\text { thinning }\end{array}$ & $118(73.3)$ & $1.5(1.3)$ \\
\hline $\begin{array}{l}\text { Fatigue/ } \\
\text { tiredness }\end{array}$ & $111(68.9)$ & $2.7(1.0)$ \\
\hline $\begin{array}{c}\text { Nausea and } \\
\text { sickness }\end{array}$ & $97(60.2)$ & $2.9(1.0)$ \\
\hline Diarrhoea & $60(37.3)$ & $2.9(1.1)$ \\
\hline Sore mouth & $36(22.4)$ & $2.8(1.2)$ \\
\hline $\begin{array}{l}\text { Increased } \\
\text { number of } \\
\text { infections }\end{array}$ & $31(19.3)$ & $2.7(1.0)$ \\
\hline Nose bleeds & $27(16.8)$ & $2.1(1.1)$ \\
\hline Anaemia & $26(16.1)$ & $2.2(0.9)$ \\
\hline $\begin{array}{l}\text { Mouth } \\
\text { ulcers }\end{array}$ & $25(15.5)$ & $2.8(1.2)$ \\
\hline Rash & $22(13.7)$ & $2.5(1.1)$ \\
\hline $\begin{array}{l}\text { Allergic } \\
\text { reaction }\end{array}$ & $15(9.3)$ & $2.8(0.7)$ \\
\hline Bruising & $10(6.2)$ & $2.0(1.1)$ \\
\hline $\begin{array}{l}\text { Bleeding } \\
\text { problems/ } \\
\text { bruising }\end{array}$ & $9(5.6)$ & $2.4(0.8)$ \\
\hline Neutropenia & $9(5.6)$ & $2.8(1.4)$ \\
\hline
\end{tabular}

${ }^{a}$ Spontaneously reported by more than one participant

(BFI), HRQoL (FACT-P) and health status (EQ-5D5L VAS) (Table 4). Key relationships among BFI, FACT-P and CTSQ scores are presented as scatterplots in Fig. 4.

\section{Experiences and Perspectives of Carers of Men with MHSPC}

The majority of carers indicated that they were satisfied with the therapy that their friend/ 


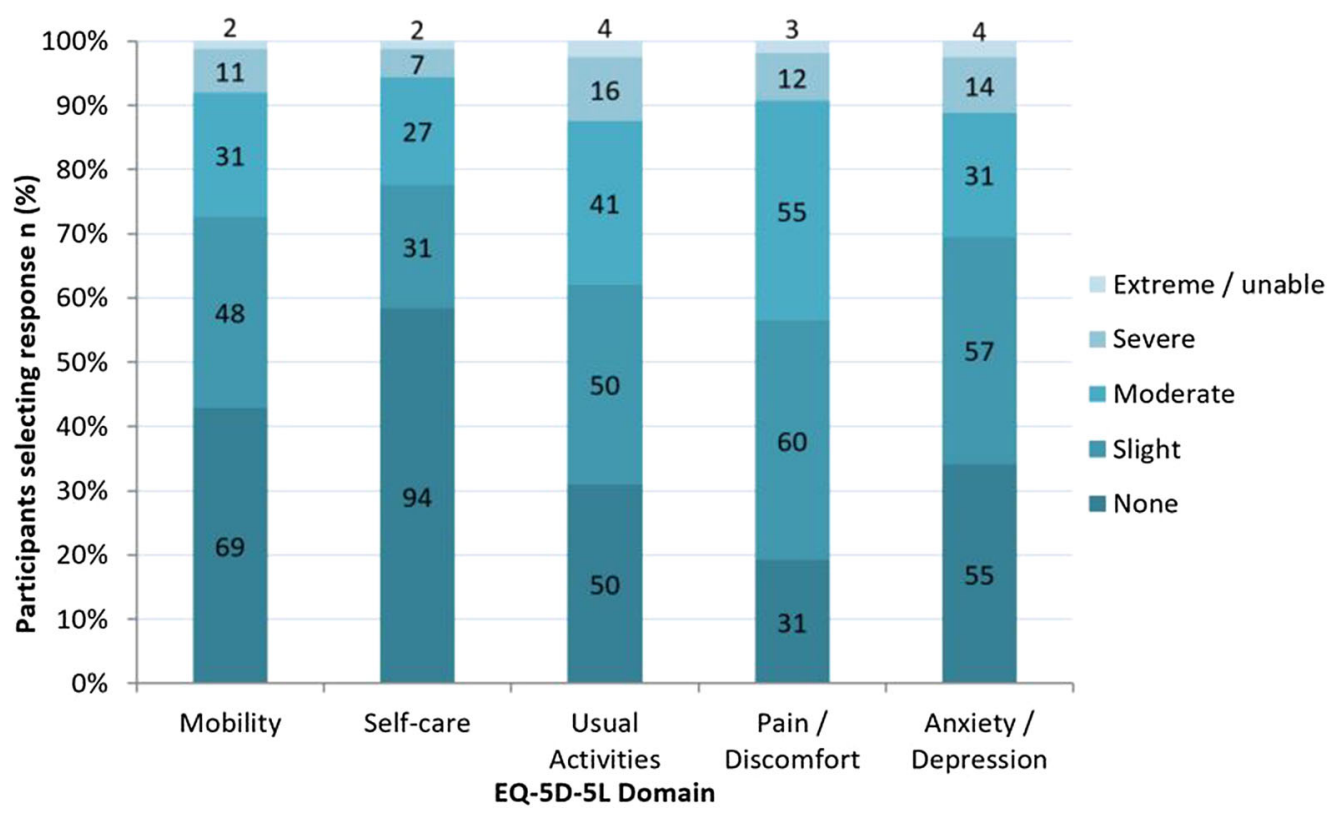

Fig. 3 EQ-5D-5L responses among men with $\operatorname{mHSPC}(n=161)$

Table 4 Pearson correlation coefficients between scores on PRO measures among men with mHSPC

\begin{tabular}{lcccccc}
\hline Variable & CTSQ ET & CTSQ FSE & CTSQ SWT & BFI & FACT-P & EQ-5D-5L index \\
\hline CTSQ ET & & & & & & \\
CTSQ FSE & 0.19 & & & & & \\
CTSQ SWT & 0.45 & 0.58 & & & & \\
BFI total & -0.07 & -0.56 & -0.46 & & & \\
FACT-P & 0.18 & 0.66 & 0.63 & -0.82 & & \\
EQ-5D-5L index & 0.04 & 0.29 & 0.23 & -0.35 & 0.46 & \\
EQ-5D-5L VAS & 0.33 & 0.47 & 0.48 & -0.50 & 0.66 & 0.37 \\
\hline
\end{tabular}

relative has received $(n=88,65.2 \%)$ and that they would be happy if their friend/relative decided to take docetaxel again $(n=90,66.7 \%)$. The majority of carers reported that the benefits of docetaxel had met or exceeded their expectations $(n=118,87.4 \%)$. When asked about side effects experienced by their friend or relative, 37 carers $(27.4 \%)$ reported that the side effects experienced had been as they expected with a further 64 carers $(47.4 \%)$ reporting that side effects were better than they had expected and $34(25.2 \%)$ reporting side effects to be worse than they expected.
Consistent with reports from men with mHSPC, the most frequently reported side effects by carers were: hair loss $(n=93,68.9 \%)$, fatigue $(n=92,68.1 \%)$ and nausea/sickness $(n=74,54.8 \%)$. Carers reported that the patients they care for were most bothered by increased number of infections, nausea/sickness, diarrhoea and mouth ulcers (Table 5).

Carer burden was explored using the BSFC. The mean score on the BSFC among the total sample of carers was 30.6 (SD 16.2). For the majority of carers $(n=105,77.0 \%)$, BSFC scores were in the none-mild range (BSFC score $=0-41)$ and indicative of carers of who are 

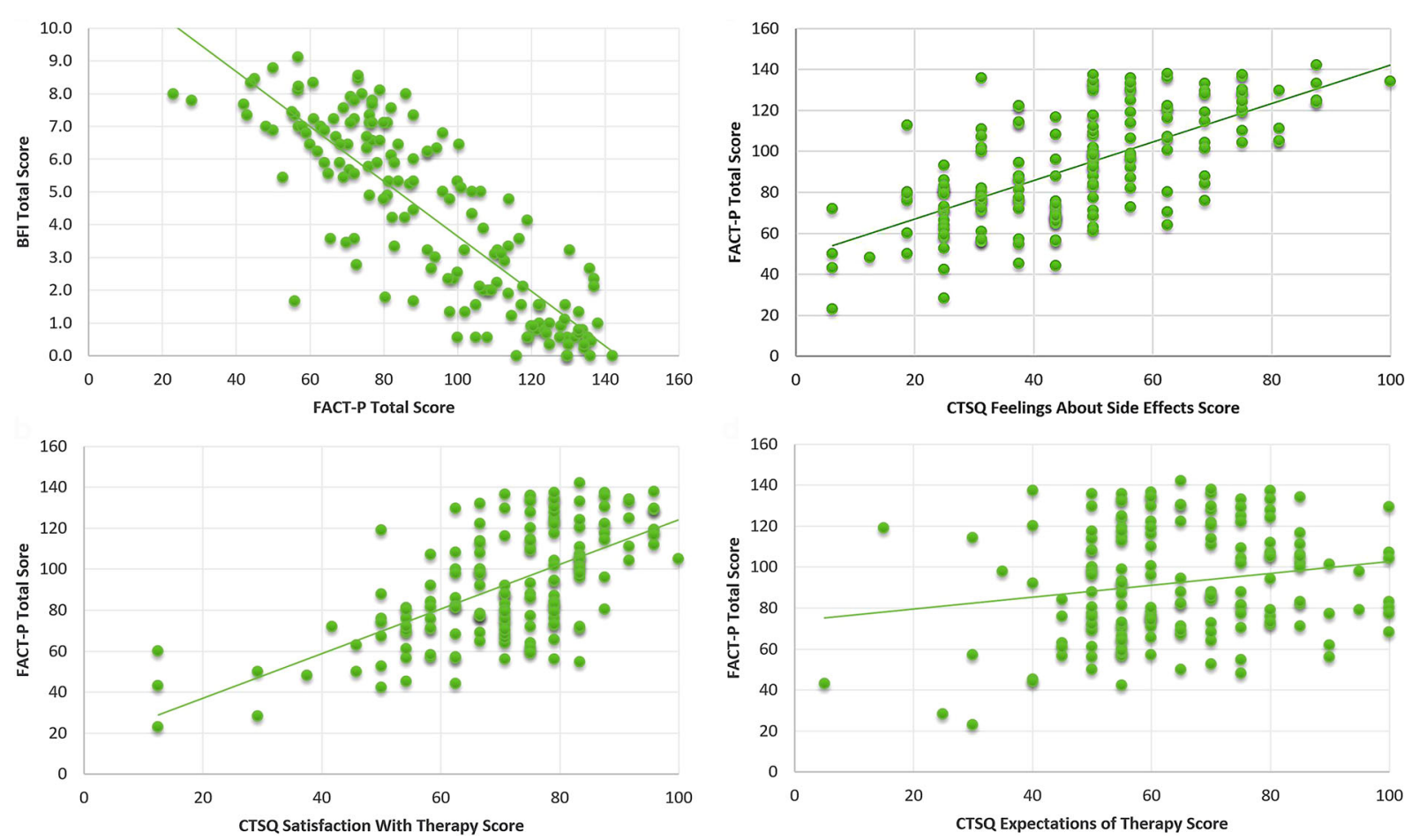

Fig. 4 Scatterplots depicting relationship among key BFI, FACT-P and CTSQ scores

not at risk of psychosomatic symptoms above and beyond what would be expected in the normal population. BSFC scores for 22 carers $(16.3 \%)$ were in the moderate range (BSFC score $=42-55)$ indicating increased risk of psychosomatic symptoms (74\% have an above average extent of physical symptoms). Only eight carers (5.9\%) had BSFC scores that were in the severe to very severe range, representing a very high risk of psychosomatic symptoms (90\% have an above average extent of physical symptoms).

\section{DISCUSSION}

The management of metastatic prostate cancer is a rapid and continuously evolving field. One of the most notable developments in recent years has been the adoption of docetaxel in combination with hormonal therapy as a firstline treatment for mHSPC. Traditionally reserved for advanced prostate cancer that is no longer responsive to hormonal therapy (i.e., castration-resistant prostate cancer), the use of a cytotoxic therapy among men with newly diagnosed mHSPC represents a significant shift in the treatment paradigm. Driving this shift has been evidence from clinical studies highlighting a survival benefit associated with the use of docetaxel among men with mHSPC [2-7]. While improving survival is a key goal of cancer therapy, there is increasing recognition that the 'quality of survival' (in terms of how patients feel and function) is also important [16].

Docetaxel is a cytotoxic chemotherapy and evidence from clinical studies highlights that, in addition to severe adverse events (e.g., neutropenia) that can lead to discontinuation of therapy, docetaxel in combination with ADT is also associated with a worsening of cancer-related symptoms such as fatigue and deficits in quality of life, which may be evident for up to 12 months following commencement of treatment [2-7]. Qualitative feedback from participants in the current study highlighted fatigue as a prominent problem among men who had received docetaxel for the treatment of $\mathrm{mHSPC}$. This was further highlighted by BFI data collected during the quantitative phase, with most 
Table 5 Treatment-related side effects among men with mHSPC as reported by carers $(n=135)$

\begin{tabular}{lll}
\hline Side effects & $\begin{array}{l}\text { Participants reporting experiencing } \\
\text { side effect, } \boldsymbol{n}(\%)\end{array}$ & $\begin{array}{l}\text { Mean participant rating of bother } \\
(\mathbf{0}-\mathbf{4} \text { : not bothered-very bothered) }\end{array}$ \\
\hline Hair loss/thinning & $93(68.9)$ & $1.4(1.2)$ \\
Fatigue/tiredness & $92(68.1)$ & $2.4(1.2)$ \\
Nausea and sickness & $74(54.8)$ & $2.7(1.1)$ \\
Diarrhoea & $49(36.3)$ & $2.7(1.0)$ \\
Sore mouth & $35(25.9)$ & $2.5(1.1)$ \\
Increased number of infections & $27(20.0)$ & $2.9(0.9)$ \\
Nose bleeds & $19(14.1)$ & $2.0(1.2)$ \\
Anaemia & $16(11.9)$ & $2.3(1.2)$ \\
Mouth ulcers & $21(15.6)$ & $2.7(1.1)$ \\
Rash & $17(12.6)$ & $1.5(1.1)$ \\
Allergic reaction & $12(8.9)$ & $2.3(1.1)$ \\
Bruising & $16(11.9)$ & $1.6(1.2)$ \\
Bleeding problems/bruising & $4(3.0)$ & $1.3(0.5)$ \\
Neutropenia & $0(0.0)$ & - \\
\hline
\end{tabular}

participants reporting feeling unusually tired or fatigued in the past week and experiencing moderate to severe fatigue. It is recognized that fatigue is a symptom with a complex aetiology than can be attributed to cancer itself, its treatment and/or a broad range of physical and psychological comorbidities [17, 18]. However, levels of fatigue reported in this study are greater than those reported at baseline and posttreatment time points in other studies among men with mHSPC. For example, in the LATITUDE study (phase III study comparing ADT with abiraterone acetate plus prednisone against ADT plus placebo) mean worst fatigue and mean average fatigue in the past $24 \mathrm{~h}$ as measured by the BFI were 2.2 and 1.8 , respectively, at the study baseline; compared with 5.1 and 4.6 in the current study [19].

Strong correlations were observed between BFI-Total and FACT-P Total scores, suggesting that fatigue is a significant driver of quality of life among men receiving docetaxel for the treatment of mHSPC. Prior investigations of docetaxel in combination with ADT
(CHAARTED) have evaluated HRQoL using FACT-P. By comparison, levels of HRQoL reported by participants in the current study are considerably lower than baseline and posttreatment evaluations in the CHAARTED study, with this finding consistent across FACT-P scores/domains. For example, mean FACT-P Total scores in the current study were 92.4 compared with 116.6-119.4 in the CHAARTED study [6].

These findings may reflect the inherent challenges of translating clinical trial data into real-world practice. Indeed, there are several confounding factors that may contribute to the observed disparities between studies including study design, sampling (e.g., presence of comorbidities), therapeutic implementation and healthcare context [20]. However, levels of HRQoL observed in this study are also lower than those observed among other real-world observations of men with mHSPC. For example, in a cross-sectional survey of 203 men with mHSPC conducted in 2014, mean EQ-5D utility index scores were reported to be 0.84 (compared 
with 0.70 in the current study). Similarly, higher FACT-P total scores (102.1 vs. 92.4) and physical well-being (21.0 vs. 17.6), emotional well-being (15.8 vs. 15.0$)$ and social well-being (18.6 vs. 13.8$)$ domain scores, which are reflective of better quality of life, were also observed in this study compared with the current study [21].

In this context, findings from the current study suggest that existing data from clinical trials may underestimate the HRQoL impact associated with use of docetaxel among men with mHSPC in a real-world setting. While there are some men with mHSPC who are functioning well on docetaxel and are generally satisfied with therapy, equally there are men who are doing less well and who are dissatisfied with therapy. Current treatment guidelines recommend that men with mHSPC be treated with ADT plus docetaxel 'provided the patient is fit enough'. Interviews with docetaxel refusers conducted as part of this study provided some insights into why men may not consider themselves suitable to receive docetaxel. However, there would appear to be little evidence available regarding the proportion of men with mHSPC who may be deemed unsuitable to receive docetaxel or indeed the parameters by which judgements regarding 'suitability' are to be made. The population of men with MHSPC is diverse in terms of both clinical and demographic characteristics. Although there is some preliminary evidence from clinical studies regarding factors that may predict both success and acceptability of docetaxel in men with mHSPC (e.g., those with high-risk disease), further research in a real-world setting is required to inform treatment decisions in practice.

Patient experience data such as those collected during this study are considered increasingly important for informing decisions in clinical development and research [22] as well as clinical practice [23]. The combination of qualitative and quantitative research techniques employed as part of a mixed methods approach and the exploration of multiple stakeholder perspectives (i.e., patients and carers) are key strengths of the current research. However, in considering the implications of findings from this study, it is necessary to acknowledge limitations that apply to this research. First, as a cross-sectional study, it is not possible to directly evaluate how docetaxel may have impacted outcomes among men with mHSPC in terms of experience of side effects and deficits in HRQoL. Further prospective observational research in which patient experiences are evaluated over time from the outset of treatment is required to better understand the consequences of docetaxel and to elucidate disparities between the experiences of men with mHSPC using docetaxel in a real-world versus a clinical trial setting. Furthermore, the sample size of the current study conforms to what is typically appropriate for qualitative research studies and for exploration of simple relationships in cross-sectional studies [24]. However, this sample size is not sufficient to explore between-group differences (based on clinical and demographic subgroups) and identification of predictors of negative or positive outcomes associated with docetaxel use. Based on the findings of this study, these are topics for further investigation.

\section{CONCLUSIONS}

The use of docetaxel alongside ADT is a welcome option for prolonging survival among men with mHSPC. Findings from this study, however, highlight that real-world experience of docetaxel may differ from that observed in clinical trials and that care must be taken to ensure that treatment options are tailored to the needs of individual patients to enhance not only how long patients survive but also the quality of that survival.

\section{ACKNOWLEDGEMENTS}

The authors would like to extend our thanks to all the men with mHSPC and carers of men with mHSPC who participated in this study.

Funding. Funding for this research, the article processing charges and the Open Access fee were provided by Janssen, High Wycombe, 
UK. All authors had full access to all the data in this study and take complete responsibility for the integrity of the data and accuracy of the data analysis.

Authorship. All named authors meet the International Committee of Medical Journal Editors (ICMJE) criteria for authorship for this article, take responsibility for the integrity of the work and have given their approval for this version to be published.

Disclosures. Tetsuro Ito is an employee of Janssen-Cilag Ltd. Laura Grant, Bryony Duckham, Amanda Ribbands and Adam Gater are employees of Adelphi who were commissioned by Janssen to conduct this research.

Compliance with Ethics and Guidelines. All procedures performed in studies involving human participants were in accordance with the ethical standards of the institutional and/or national research committee and with the 1964 Helsinki Declaration and its later amendments or comparable ethical standards. Informed consent was obtained from all individual participants included in the study.

Data Availability. The data sets generated during and/or analysed during the current study are available from the corresponding author on reasonable request.

Open Access. This article is distributed under the terms of the Creative Commons Attribution-NonCommercial 4.0 International License (http://creativecommons.org/licenses/ by-nc/4.0/), which permits any noncommercial use, distribution, and reproduction in any medium, provided you give appropriate credit to the original author(s) and the source, provide a link to the Creative Commons license, and indicate if changes were made.

\section{REFERENCES}

1. Cancer Research UK. Prostate cancer incidence by stage at diagnosis. http://www.cancerresearchuk. org/health-professional/cancer-statistics/statistics- by-cancer-type/prostate-cancer/incidence\#ref-8. Accessed 20 Aug 2018.

2. Puente J, Grande E, Medina A, Maroto P, Lainez N, Arranz JA. Docetaxel in prostate cancer: a familiar face as the new standard in a hormone-sensitive setting. Ther Adv Med Oncol. 2017;9:307-18.

3. Tucci M, Bertaglia V, Vignani F, et al. Addition of docetaxel to androgen deprivation therapy for patients with hormone-sensitive metastatic prostate cancer: a systematic review and meta-analysis. Eur Urol. 2016;69(4):563-73.

4. James ND, Spears MR, Clarke NW, et al. Failure-free survival and radiotherapy in patients with newly diagnosed nonmetastatic prostate cancer: data from patients in the control arm of the STAMPEDE trial. JAMA Oncol. 2016;2(3):348-57.

5. James ND, Sydes MR, Clarke NW, et al. Addition of docetaxel, zoledronic acid, or both to first-line longterm hormone therapy in prostate cancer (STAMPEDE): survival results from an adaptive, multiarm, multistage, platform randomised controlled trial. Lancet. 2016;387(10024):1163-77.

6. Sweeney CJ, Chen Y-H, Carducci M, et al. Chemohormonal therapy in metastatic hormone-sensitive prostate cancer. N Engl J Med. 2015;373(8):737-46.

7. Gravis G, Fizazi K, Joly F, et al. Androgen-deprivation therapy alone or with docetaxel in non-castrate metastatic prostate cancer (GETUG-AFU 15): a randomised, open-label, phase 3 trial. Lancet Oncol. 2013;14(2):149-58.

8. Parker C, Gillessen S, Heidenreich A, Horwich A, Committee EG. Cancer of the prostate: ESMO Clinical Practice Guidelines for diagnosis, treatment and follow-up. Ann Oncol Off J Eur Soc Med Oncol. 2015;26(Suppl 5):v69-77.

9. Mottet N, Bellmunt J, Briers E, et al. EAU-ESTROESUR-SIOG Guidelines on prostate cancer: part 1: screening, diagnosis, and local treatment with curative intent. Eur Urol. 2017;71(4):618-29.

10. Trask PC, Tellefsen C, Espindle D, Getter C, Hsu MA. Psychometric validation of the cancer therapy satisfaction questionnaire. Value Health. 2008;11(4): 669-79.

11. Shahid A, Wilkinson K, Marcu S, Shapiro CM. Brief Fatigue Inventory. STOP, THAT and one hundred other sleep scales. New York: Springer; 2011. p. 75-7.

12. Esper P, Mo F, Chodak G, Sinner M, Cella D, Pienta KJ. Measuring quality of life in men with prostate cancer using the functional assessment of cancer 
therapy-prostate instrument. Urology. 1997;50(6): 920-8.

13. Rabin R, Charro FD. EQ-SD: a measure of health status from the EuroQol Group. Ann Med. 2001;33(5):337-43.

14. Gräßel E, Chiu T, Oliver R. Development and validation of the Burden Scale for Family Caregivers (BSFC). Toronto: COTA Comprehensive Rehabilitation and Mental Health Services; 2003.

15. Szende A, Janssen B. Cross-Country analysis of EQ5D data. In: Szende A, Janssen B, Cabases J, editors. Self-reported population health: an international perspective based on EQ-5D. Dordrecht: Springer; 2014. p. 31-6.

16. Fallowfield L, Nadler E, Gilloteau I, et al. Quality of survival: a new concept framework to assess the quality of prolonged life in cancer. Expert Rev Qual Life Cancer Care. 2017;2(4):225-32.

17. Charalambous A, Kouta C. Cancer related fatigue and quality of life in patients with advanced prostate cancer undergoing chemotherapy. Biomed Res Int. 2016;2016:3989286.

18. Bergin ART, Hovey E, Lloyd A, et al. Docetaxel-related fatigue in men with metastatic prostate cancer: a descriptive analysis. Support Care Cancer. 2017;25(9):2871-9.

19. Fizazi K, Chi KN. Abiraterone in metastatic prostate cancer. N Engl J Med. 2017;377(17):1697-8.
20. Nussbaum N, George DJ, Abernethy AP, et al. Patient experience in the treatment of metastatic castration-resistant prostate cancer: state of the science. Prostate Cancer Prostatic Dis. 2016;19(2):111-21.

21. Lin HM, Zhu Y, Rider A, Murray G, Roughley A, Piercy J. Impact of staging and hormone sensitivity on quality of life in us prostate cancer patients. Value Health. 2015;18(7):A468.

22. US Department of Health and Human Services Food and Drug Administration Center for Drug Evaluation and Research (CDER), Center for Biologics Evaluation and Research (CBER). Patient-Focused Drug Development: Collecting Comprehensive and Representative Input Guidance for Industry, Food and Drug Administration Staff, and Other Stakeholders. Draft Guidance. June 2018. https://www. fda.gov/downloads/Drugs/GuidanceCompliance RegulatoryInformation/Guidances/UCM610442. pdf. Accessed June 2018.

23. Doyle C, Lennox L, Bell D. A systematic review of evidence on the links between patient experience and clinical safety and effectiveness. BMJ Open. 2013;3(1):e001570.

24. VanVoorhis CW, Morgan BL. Understanding power and rules of thumb for determining sample sizes. Tutor Quant Methods Psychol. 2007;3(2):43-50. 